\title{
Groups generated by two bicyclic units in integral group rings
}

Eric Jespers

Dep. Mathematics

Vrije Universiteit Brussel

Pleinlaan 2

1050 Brussel, Belgium

efjesper@vub.ac.be ngel del Ro

Dep. Matemáticas

Universidad de Murcia

Campus de Espinardo

30100 Murcia, Spain

adelrio@fcu.um.es
Manuel Ruiz

Dep. Mtodos Cuantitativos e Informticos

Universidad Politcnica de Cartagena

Paseo Alfonso XIII, 50

30.203 Cartagena, Spain

Manuel.Ruiz@upct.es

Proposed Running title: Groups generated by two bicyclics 


\title{
Groups generated by two bicyclic units in integral group rings
}

\author{
Eric Jespers, ngel del Ro and Manuel Ruiz *
}

\section{Introduction}

In [5] Ritter and Sehgal introduced the following units, called the bicylic units, in the unit group $\mathcal{U}(\mathbb{Z} G)$ of the integral group ring $\mathbb{Z} G$ of a finite group $G$ :

$$
\beta_{a, g}=1+(1-g) a \widehat{g}, \quad \gamma_{a, g}=1+\widehat{g} a(1-g),
$$

where $a, g \in G$ and $\widehat{g}$ is the sum of all the elements in the cyclic group $\langle g\rangle$.

It has been shown that these units generate a large part of the unit group of $\mathbb{Z} G$. Indeed, for most finite groups $G$, the bicyclic units together with the Bass cyclic units generate a subgroup of finite index in $\mathcal{U}(\mathbb{Z} G)[3,6]$. The Bass cyclic units are only needed to cover a subgroup of finite index in the centre and the group $B$ generated by the bicyclic units contains a subgroup of finite index in a maximal $\mathbb{Z}$-order of each non-commutative simple image $M_{n}(D)$ of the rational group algebra $\mathbb{Q} G$. In particular, if $n>1$, then $B$ contains a subgroup of finite index in $\operatorname{SL}_{n}(\mathcal{O})$, where $\mathcal{O}$ is a maximal order in $D$; and hence $B$ contains free subgroups of rank two. A next step in determining the structure of $\mathcal{U}(\mathbb{Z} G)$ is to investigate relations among the discovered generators. Presently this is beyond reach. Hence a more realistic goal is to study the structure of the group generated by two bicyclic units. In [4] Marciniak and Sehgal proved that if $\beta_{a, g}$ is a non trivial unit in $\mathbb{Z} G$ (here $G$ is not necessarily finite) then the group $\left\langle\beta_{a, g}, \gamma_{a^{-1}, g^{-1}}\right\rangle$ is free of rank 2. Clearly, bicyclic units are of the form $1+a$ with $a^{2}=0$. Salwa, in [7], used the ideas of Marciniak and Sehgal to prove that if $x$ and $y$ are two elements of an additively torsion-free ring such that $x^{2}=y^{2}=0$ and $x y$ is not nilpotent then $\left\langle(1+x)^{m},(1+y)^{m}\right\rangle$ is free of rank 2 for some positive integer $m$. In particular, if $b_{1}$ and $b_{2}$ are two bicyclic units and $\left(b_{1}-1\right)\left(b_{2}-1\right)$ is not nilpotent, then $\left\langle b_{1}^{m}, b_{2}^{m}\right\rangle$ is free of rank 2 for some positive integer $m$. In this paper we investigate the minimum positive integer $m$ so that $\left\langle b_{1}^{m}, b_{2}^{m}\right\rangle$ is free provided that $b_{1}$ and $b_{2}$ are two bicyclic units so that $\left(b_{1}-1\right)\left(b_{2}-1\right)$ is not nilpotent. We prove the following theorem which indicates that if $b_{1}$ and $b_{2}$ are of the same type then frequently $m=1$.

Theorem 1.1 Let $b_{1}=\beta_{x, g}$ and $b_{2}=\beta_{y, h}$ be two bicyclic units of the same type in the dihedral group

$$
D_{n}=\left\langle a, b \mid a^{n}=b^{2}=1, b a=a^{-1} b\right\rangle
$$

so that $\langle y, h\rangle \subseteq\langle x, g\rangle$. Then $\left\langle b_{1}, b_{2}\right\rangle$ is either torsionfree abelian or free of rank 2 .

As a consequence one obtains the following.

Corollary 1.2 If $p$ is prime and $b_{1}$ and $b_{2}$ are two bicyclic units of the same type of the dihedral group $D_{p}$ then $\left\langle b_{1}, b_{2}\right\rangle$ is either torsion free abelian or free of rank 2.

${ }^{*}$ The first author has been partially supported by the Onderzoeksraad of Vrije Universiteit Brussel and the Fonds voor Wetenschappelijk Onderzoek (Vlaanderen) and the second by the D.G.I. of Spain and Fundacin Sneca of Murcia. 
We prove Theorem 1.1 in Section 3. Before that we revisit Salwas's Theorem in Section 2 with the following two aims: first to present the tools needed in our proof of Theorem 1.1 and second to complement Salwa's result with the case that $x y$ is nilpotent. Namely we prove the following

Theorem 1.3 Let $K$ be a subfield of $\mathbb{C}$ and $R$ a finite dimensional $K$-algebra. Suppose $a, b$ are two elements of $R$ such that $a^{2}=b^{2}=0$. The following properties hold:

1. if $a b$ is nilpotent, then $\langle 1+a, 1+b\rangle$ is nilpotent;

2. if ab is not nilpotent then there is a positive integer $m$ so that $\left\langle 1+a,(1+b)^{m}\right\rangle$ is free of rank 2 .

We finish the paper with some comments on the cases not included in Theorem 1.1. In particular we relate the problem for arbitrary dihedral groups to an open problem on free points for groups generated by a pair of $2 \times 2$-matrices (see for example the recent work of Bamberg [1]).

\section{Nilpotent-Free dichotomy}

In this section we prove Theorem 1.3. Many of the ideas are already in [7] where the second statement of the theorem is proved. Also, under some conditions (see the last part of Lemma 2.3) on a trace map, it is shown that $m=1$. For completeness' sake we give a selfcontained proof.

Throughout this section $K$ is a subfield of $\mathbb{C}, R$ is a finite dimensional $K$-algebra and $a$ and $b$ are two elements of $R$ so that $a^{2}=b^{2}=0$. In order to prove Theorem 1.3 one may assume that $R=K[a, b]$. Let $J(R)$ denote the Jacobson radical of $R$. So $R / J(R)=\sum_{i=1}^{k} M_{n_{i}}\left(D_{i}\right)$, where $D_{i}$ is a division $K$-algebra for every $i$.

If $x$ is a real number then $\lfloor x\rfloor$ (resp. $\lceil x\rceil$ ) denotes the greatest (resp. smallest) integer not larger (resp. not smaller) than $x$.

Lemma 2.1 For every $i=1, \ldots, k$ one has $n_{i} \leq 2$ and $D_{i}$ is a field. Furthermore, if $n_{i}=1$ then $D_{i}=K$.

Proof. Let $A=\mathbb{C}[a, b]=\mathbb{C} \otimes_{K} K[a, b]=\mathbb{C} \otimes_{K} R$. First we prove that every simple quotient of $A / J(A)$ is of the form $M_{m}(\mathbb{C})$ with $m \leq 2$. We may assume without loss of generality that $A$ is simple, so that $A=M_{m}(\mathbb{C})$ for some positive integer $m$ and we identify $A$ with the endomorphism ring of an $m$-dimensional complex vector space. Let $B=\mathbb{C}[a b]$. Then $A=B+B a+b B+b B a$ and hence $\operatorname{dim}_{\mathbb{C}} A \leq 4 \operatorname{dim}_{\mathbb{C}} B$. Since $a^{2}=0$ we have $\operatorname{Im} a \subseteq$ Ker $a$ and hence $2 \operatorname{dim}_{\mathbb{C}} \operatorname{Im} a \leq m$. Consequently, $\operatorname{dim}_{\mathbb{C}} \operatorname{Im} a b \leq \operatorname{dim}_{\mathbb{C}} \operatorname{Im} a \leq\left\lfloor\frac{m}{2}\right\rfloor$. The Cayley-Hamilton Theorem then implies that $a b$ satisfies a polynomial identity of degree at most $\left\lfloor\frac{m}{2}\right\rfloor+1$. Hence $\operatorname{dim}_{\mathbb{C}} B \leq\left\lfloor\frac{m}{2}\right\rfloor+1$ and thus $m^{2}=\operatorname{dim}_{\mathbb{C}} A \leq 4\left(\left\lfloor\frac{m}{2}\right\rfloor+1\right)$. Consequently $\left(\frac{m}{2}\right)^{2} \leq\left\lfloor\frac{m}{2}\right\rfloor+1$, and therefore $m \leq 2$.

Clearly, both $J(R)$ and $J(A)$ are nilpotent. As $A$ is a central extension of $R$ we know that $J(R)=J(A) \cap R$. So we consider $R / J(R)$ as a subring of $A / J(A)$.

Let $S=M_{n}(D)$, with $D$ a division ring, be a simple quotient of $R$. By the above $S$ is embedded in a simple quotient $M_{m}(\mathbb{C})$ of $A$, with $m \leq 2$. Hence every complete set of orthogonal idempotents of $S$ has at most 2 elements. So $n \leq 2$. Furthermore, if $n=2$, then $m=2$ and $D$ being the double centralizer of a set of a primitive idempotents yields that $D \subseteq \mathbb{C}$. So, in this case, $D$ is a field. On the other hand, if $m=1$, then the natural images $\bar{a}$ and $\bar{b}$ in $S=D$ are zero. Hence $S=K[\bar{a}, \bar{b}]=K$. This proves the result. 
Lemma 2.2 Let $A=M_{2}(D)$ where $D$ is a division ring and $a, b \in A$ so that $a^{2}=b^{2}=0$. If $a b$ is nilpotent then $a b=b a=0$.

Proof. Again, we identify $A$ with the endomorphism ring of a two dimensional vector space $V$ over $D$. If $a b \neq 0$ then Ker $a b=\operatorname{Ker} b=\operatorname{Im} b$ and $\operatorname{Im} a b=\operatorname{Im} a=\operatorname{Ker} a$. Let $0 \neq v_{1} \in \operatorname{Im} a$ and $v_{2} \in V$ so that $a\left(v_{2}\right)=v_{1}$. Then $V=\left\langle v_{1}, v_{2}\right\rangle$. Furthermore $(a b)\left(v_{1}\right)=\lambda v_{1}$ for some $\lambda \in K$. Since $a b$ is nilpotent, $\lambda=0$. Thus $(a b)^{2}=0$ and therefore Ker $b=\operatorname{Ker} a b=\operatorname{Im} a b=\operatorname{Im} a$. We conclude that $a b=0$, a contradiction.

Lemma 2.2 implies that if $a b$ is nilpotent, then $R a+J(R)$ and $R b+J(R)$ are nilpotent left ideals of $R$. So $a, b \in J(R)$. Hence $\langle 1+a, 1+b\rangle$ is contained in the (multiplicative) nilpotent group $1+J(R)$. This proves statement 1 of Theorem 1.3 .

To prove the second statement of Theorem 1.1, first recall that from from Sanov's Theorem [8] and a change of basis argument it is easy to deduce that if $z$ and $w$ are two complex numbers so that $|z w| \geq 4$, then the following two matrices generate a free group of rank 2 :

$$
\left(\begin{array}{ll}
1 & z \\
0 & 1
\end{array}\right), \quad\left(\begin{array}{cc}
1 & 0 \\
w & 1
\end{array}\right)
$$

If $a b$ is not nilpotent, then $\rho(a b)$ is not nilpotent where $\rho: R \rightarrow M_{n_{i}}\left(D_{i}\right)$ is an epimorphism for some $i=1, \ldots, k$ with $n_{i}=2$. Statement 2 of Theorem 1.3 then follows from the following Lemma.

Lemma 2.3 Let $A=M_{2}(K)$, where $K$ is a subfield of $\mathbb{C}$ and $a, b \in A$ so that $a^{2}=b^{2}=0$ and $a b$ is not nilpotent. Then $\langle 1+a, 1+m b\rangle$ is a free group for some positive integer $m$. If, moreover, $|\operatorname{tr}(a b)| \geq 4$, then $\langle 1+a, 1+b\rangle$ is a free group.

Proof. Again we identify $A$ with the endomorphism ring of the two dimensional $K$-vector space $K^{2}$. Since $a b$ is not nilpotent of rank 1 , after a change of basis in $K^{2}$ one may assume that

$$
a b=\left(\begin{array}{cc}
\lambda & 0 \\
0 & 0
\end{array}\right)
$$

for some nonzero $\lambda \in K$.Hence, as $\operatorname{Im} a=\operatorname{Im} a b$ and Ker $b=\operatorname{Ker} a b$ one has that

$$
a=\left(\begin{array}{cc}
p & r \\
0 & 0
\end{array}\right) \quad \text { and } \quad b=\left(\begin{array}{cc}
q & 0 \\
s & 0
\end{array}\right)
$$

for some $p, q, r, s \in K$. Because $a$ and $b$ are nonzero nilpotent elements, $p=0=q$ and $r \neq 0$ and $s \neq 0$. Thus

$$
1+a=\left(\begin{array}{cc}
1 & r \\
0 & 1
\end{array}\right) \quad \text { and } \quad 1+b=\left(\begin{array}{ll}
1 & 0 \\
s & 1
\end{array}\right) .
$$

By Sanov's Theorem $\langle 1+a, 1+m b\rangle$ is a free group for $m=\left\lceil\frac{4}{|r s|}\right\rceil$. If $|\operatorname{tr}(a b)| \geq 4$, then $|r s| \geq 4$ and hence $m=1$.

Proposition 2.4 Let $A$ be a $\mathbb{Q}$-algebra which is a direct product of division rings and $2 \times 2$-matrix rings over subfields of $\mathbb{C}$. Suppose $a, b \in A$ are such that $a^{2}=b^{2}=0$. The following properties hold:

1. if ab is nilpotent, then $\langle 1+a, 1+b\rangle$ is torsionfree abelian; 
2. if ab is not nilpotent, then there exists an integer $m$ so that $\langle 1+a, 1+m b\rangle$ is free of rank 2. Moreover if $|\operatorname{tr}(\rho(a b))| \geq 4$, for some projection $\rho: A \rightarrow M_{2}(K)$ onto a simple quotient of $A$, then $\langle 1+a, 1+b\rangle$ is free of rank 2 .

Proof. Everything is an immediate consequence of Lemma 2.2 and Lemma 2.3 except the torsionfreeness of the abelian case.

Assume that $u=(1+a)^{k}(1+b)^{l}$ is a periodic element of order $m$ in the abelian group $\langle 1+a, 1+b\rangle$ (with $k, l \in \mathbb{Z}$ ). Then, $1+k m a=1-l m b$ and so $k a=-l b$. Hence, $(1+a)^{k}=1+k a=1-l b=(1+b)^{-l}$ and thus $u=1$.

\section{Bicyclic units}

In this section we prove Theorem 1.1. Let $b_{1}=\beta_{x, g}$ and $b_{2}=\beta_{y, h}$ be two bicyclic units of $D_{n}$ so that $\langle y, h\rangle \subseteq\langle x, g\rangle$. Note that $\mathbb{Q} D_{n}$ satisfies the conditions of Proposition 2.4. So either $\left\langle b_{1}, b_{2}\right\rangle$ is torsionfree abelian or $\left\langle b_{1}, b_{2}^{k}\right\rangle$ is free of rank 2 for some $k \geq 1$. We have to prove that in the former case one can take $k=1$. For that we make use once more of Proposition 2.4 and we only have to prove that if $b_{1}$ and $b_{2}$ do not commute then there exists an irreducible complex character $\chi$ of degree 2 of $D_{n}$ so that $|\chi(\alpha)| \geq 4$, where $\alpha=\left(b_{1}-1\right)\left(b_{2}-1\right)$.

So in the remainder we assume that $b_{1}$ and $b_{2}$ do not commute. This imply that both $g$ and $h$ do not belong to $\langle a\rangle$ and hence $\langle x, g\rangle$ is a dihedral group. Therefore one may assume without loss of generality that $D_{n}=\langle x, g\rangle$. Since $\beta_{a^{i} b, a^{j} b}=\beta_{a^{i-j}, a^{j} b}$, without loss of generality, we may assume that $D_{n}=\langle x, g\rangle, x, y \in\langle a\rangle$ and changing generators if necessary, $x=a$ and $g=b$. Summarizing $b_{1}=\beta_{a, b}$ and $b_{2}=\beta_{a^{i}, a^{j} b}$ for some $1 \leq i, j<n$ and $2 i \neq n$.

The non-linear irreducible complex characters of $D_{n}$ are all maps $\chi_{k}$, with $1 \leq k<\frac{n}{2}$, given by

$$
\chi_{k}\left(a^{t}\right)=\xi^{t}+\xi^{-t}, \quad \chi_{k}\left(a^{t} b\right)=0
$$

for every $0 \leq t<n$, where $\xi$ denotes an $n$-th root of unity.

For every $m \in \mathbb{Z}$ we denote:

$$
\eta_{m}=\xi^{m}+\xi^{-m}=2 \cos \frac{2 \pi m}{n}, \quad \nu_{m}=\xi^{m}-\xi^{-m}=2 \sin \frac{2 \pi m}{n} .
$$

The following formulae are easily verified:

$$
\begin{aligned}
& \eta_{n} \eta_{m}=\eta_{n+m}+\eta_{n-m} \\
& \nu_{n} \nu_{m}=\eta_{n+m}-\eta_{n-m} \\
& \nu_{m} \eta_{n}=\nu_{m+n}+\nu_{m-n}
\end{aligned}
$$

Then

$$
\begin{aligned}
\alpha= & a^{i+1}-a^{i-1}+a^{-i-1}-a^{1-i}+a^{1-i-j}+a^{i-1-j}-a^{1+i-j}-a^{-1-i-j}+ \\
& \left(a^{1-i}+a^{i-1}-a^{1+i}-a^{-1-i}+a^{1+i+j}+a^{-1-i+j}-a^{-1+i+j}-a^{1-i+j}\right) b
\end{aligned}
$$

and hence

$$
\begin{aligned}
\chi_{k}(\alpha) & =\eta_{(i+1) k}-\eta_{(i-1) k}+\eta_{(-1-i) k}-\eta_{(1-i) k}+\eta_{(1-i-j) k}+\eta_{(i-1-j) k}-\eta_{(1+i-j) k}-\eta_{(-1-i-j) k} \\
& =2 \nu_{k} \nu_{i k}+\eta_{-j k}\left(\nu_{(1-i) k}-\nu_{(1+i) k}\right)=\nu_{k} \nu_{i k}\left(2-\eta_{j k}\right) \\
& =8 \sin \frac{2 \pi k}{n} \sin \frac{2 \pi i k}{n}\left(1-\cos \frac{2 \pi j k}{n}\right)=16 \sin \frac{2 \pi k}{n} \sin \frac{2 \pi i k}{n} \sin ^{2} \frac{\pi j k}{n} .
\end{aligned}
$$

The existence of a character with the required properties then follows from the following Lemma. The remainder of the section is devoted to its proof. Unfortunately the proof is longer than one would like. On the other hand the remarks given in Section 4 indicates that the inequality of the Lemma is quite elusive. 
Lemma 3.1 If $i, j, n$ are positive integers such that $1 \leq i, j<n, n \geq 3$ and $(n, i, j) \neq(6,3,1)$ then there is $k \in \mathbb{Z}$ such that

$$
\left|\sin \frac{2 \pi k}{n} \sin \frac{\pi i k}{n} \sin ^{2} \frac{\pi j k}{n}\right| \geq \frac{1}{4}
$$

Proof. For every integer $a \in \mathbb{R}$ let $s_{a}=\frac{2 \pi a}{n}$ and $f_{a}: \mathbb{R} \rightarrow \mathbb{R} / 2 \pi \mathbb{Z}=S$ be the map defined by $f_{a}(x)=s_{a} x+2 \pi \mathbb{Z}$.

Let $i, j, n$ be integers satisfying the conditions of the Lemma. Without loss of generality we may assume that $i, j \leq \frac{n}{2}$, so that $s_{i}, s_{j} \leq \pi$. Let $f=f_{i, j}:[0, n) \rightarrow T=S^{2}$ be defined by $f(x)=\left(f_{i}(x), f_{j}(x)\right)$. The image of $f$ is a spiral on the torus $T$.

We identify $S$ with $[0,2 \pi)$ and $T$ with the square $[0,2 \pi)^{2}$, so that we consider $f_{a}$ as a map $\mathbb{R} \rightarrow[0,2 \pi)$. Let $D$ denote the diagonal of $T$ and $V=\left[\frac{\pi}{2}, \frac{3 \pi}{2}\right]^{2}$.
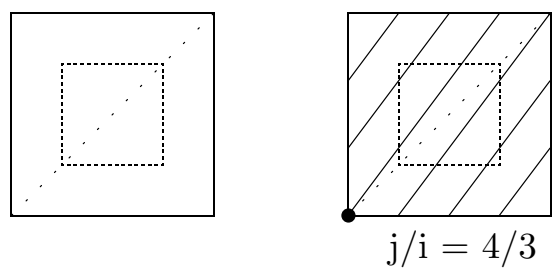

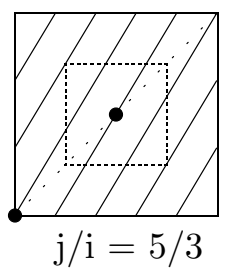

Figure 1
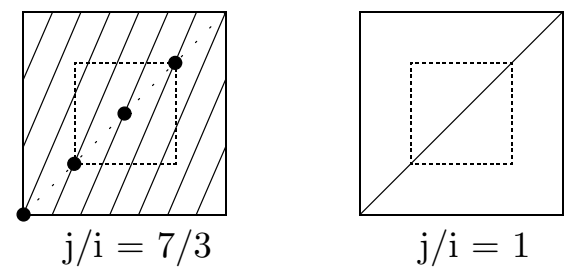

The first of the pictures in Figure 1 represents $T$ (the big square), $D$ (the dotted line) and $V$ (the small square). The other pictures include the image of $f$ for different values of $j / i$.

Let

$$
W=\left[\frac{n}{8}, \frac{3 n}{8}\right], \quad W_{1}=\left[\left\lceil\frac{n}{8}\right\rceil,\left\lfloor\frac{3 n}{8}\right\rfloor\right], \quad X=f^{-1}(D)
$$

and consider the following four conditions:

(1) There are $k_{1}, k_{2} \in \mathbb{Z}$ such that $\left|\sin f_{i}\left(k_{1}\right) \sin f_{i}\left(k_{1} / 2\right) \sin ^{2} f_{j}\left(k_{1} / 2\right)\right| \geq \frac{1}{4}$ and $\left|\sin f_{i}\left(k_{1}\right) \sin ^{2} f_{i}\left(k_{1} / 2\right) \sin f_{j}\left(k_{1} / 2\right)\right| \geq \frac{1}{4}$.

(2) There are $k_{1}, k_{2} \in \mathbb{Z} \cap W$ such that $\left|\sin f_{i}\left(k_{1} / 2\right) \sin ^{2} f_{j}\left(k_{1} / 2\right)\right| \geq \frac{\sqrt{2}}{4}$ and $\left|\sin ^{2} f_{i}\left(k_{2} / 2\right) \sin f_{j}\left(k_{2} / 2\right)\right| \geq \frac{\sqrt{2}}{4}$.

(3) $f(\mathbb{Z} \cap W) \cap V \neq \emptyset$.

(4) $f\left(W_{1}\right) \cap D \cap V \neq \emptyset$.

Condition (1) is equivalent to the statement of the lemma. This condition is introduced to get symmetry in the roles of $i$ and $j$. Clearly (3) implies (2) and (2) implies (1). Now we prove that (4) implies (3). Let $t \in W_{1}$ be such that $f(t) \in V \cap D$. Then there is an integer $l$ such that $l \leq t \leq l+1$ and $l, l+1 \in W_{1} \subseteq W$. Assume that $i \leq j$. Since $s_{j} \leq \pi$ and $\frac{\pi}{2} \leq f_{j}(t) \leq \frac{3 \pi}{2}$, then either $\frac{\pi}{2} \leq f_{j}(l) \leq f_{i}(l) \leq f_{i}(t) \leq \frac{3 \pi}{2}$ or $\frac{\pi}{2} \leq f_{i}(t) \leq f_{i}(l+1) \leq f_{j}(l+1) \leq \frac{3 \pi}{2}$. Thus (3) holds.

We now argue by contradiction. So assume the Lemma is not true and thus that the conditions (1) to (4) do not hold. Hence, from now on we work under the following assumptions:

(C1) either, for every $k \in \mathbb{Z},\left|\sin f_{1}(k) \sin f_{i}(k / 2) \sin ^{2} f_{j}(k / 2)\right|<\frac{1}{4}$, or, for every $k \in \mathbb{Z}$, $\left|\sin f_{1}(k) \sin ^{2} f_{i}(k / 2) \sin f_{j}(k / 2)\right|<\frac{1}{4}$. 
(C2) either for every $k \in \mathbb{Z},\left|\sin f_{i}(k / 2) \sin ^{2} f_{j}(k / 2)\right|<\frac{\sqrt{2}}{4}$, or for every $k \in \mathbb{Z}$, $\left|\sin ^{2} f_{i}(k / 2) \sin f_{j}(k / 2)\right|<\frac{\sqrt{2}}{4}$.

(C3) $f(\mathbb{Z} \cap W) \cap V=\emptyset$.

(C4) $f\left(W_{1}\right) \cap D \cap V=\emptyset$ or equivalently $W_{1} \cap X \cap f^{-1}(V) \neq \emptyset$.

An exhaustive computer search shows that the only triple of positive integers $(n, i, j)$ with $n \leq 200$ and $i, j \leq \frac{n}{2}$ for which there is not an integer $k$ satisfying $(3.1)$ is $(n, i, j)=(6,3,1)$; precisely the case excluded in the statement of the Lemma. So we assume $n>200$. Note that because of condition $(\mathrm{C} 4)$ and the fact that $s_{i}, s_{j} \leq \pi$ we also easily deduce that $i \neq j$. Moreover, because of the symmetry of the roles of $i$ and $j$, we may also assume that $i<j$.

We introduce the following notation:

$$
\begin{aligned}
m & =\operatorname{gcd}(i, j)=\left|f^{-1}(0,0)\right| \\
v & =\frac{j-i}{m}=|D \cap \operatorname{Im} f| \\
\alpha & =\frac{n}{m v} \\
\theta & =f_{i}(\alpha)\left(=f_{j}(\alpha)\right) .
\end{aligned}
$$

Then

$$
\begin{aligned}
X & =\{k \alpha: k=0,1, \ldots, m v-1\} \text { and } \\
f(X) & =D \cap \operatorname{Im} f=\left\{\left(\frac{2 \pi k}{v}, \frac{2 \pi k}{v}\right): k=0,1, \ldots, v-1\right\}
\end{aligned}
$$

It is clear that $f(X)$ is a cyclic subgroup of $D \cap T$ of order $v$ and $(\theta, \theta)$ is a generator of $f(X)$. The dots in the previous picture represent the elements of $f(X)$.

Since $f(X) \cap V=\operatorname{Im} f \cap V \cap D$, condition (C4) implies that

(C5) If $I$ is an interval so that $f(I)=\operatorname{Im} f$ then $f(X) \cap V \subseteq f\left(\left(I \backslash W_{1}\right) \cap X\right)$.

Since $\alpha=\frac{n}{j-i}>2$, every interval of length $\leq 2$ contains at most one element of $X$ and therefore condition (C4) implies

$$
\left|\left[\frac{n}{8},\left[\frac{n}{8}\right\rceil\right] \cap X\right| \leq 1, \quad\left|\left[\left\lfloor\frac{3 n}{8}\right\rfloor, \frac{3 n}{8}\right] \cap X\right| \leq 1 \quad \text { and hence } \quad\left|W \cap X \cap f^{-1}(V)\right| \leq 2 .
$$

We consider separately the mutually exclusive cases (1) $v \neq 1$ and $m v \neq 2,(2)$ and $v=1$ and $m \geq 3$ and $(3) m v \leq 2$.

Case 1: $v \neq 1$ and $m v \neq 2$. First we show that $m \leq 4$. Note that

$$
|f(X) \cap V|=\left\{\begin{array}{llll}
\frac{v}{2}+1 & \text { if } & v \equiv 0 & \bmod 4 \\
\frac{v}{2} & \text { if } & v \equiv 2 & \bmod 4 \\
\frac{v+1}{2} & \text { if } & v \equiv 3 \bmod 4 \\
\frac{v-1}{2} & \text { if } & v \equiv 1 & \bmod 4
\end{array}\right.
$$

which is different from 0 . Let $I$ be the interval centred at $\frac{n}{4}$ and of length $\frac{n}{m}$. As $f(I)=\operatorname{Im} f$, condition (C5) implies that $I$ is not contained in $W_{1}$ and this implies that $m \leq 4$.

Second we show that $m \neq 4$. So, suppose the contrary, that is assume $v \neq 1$ and $m=4$. Because $W$ has length $\frac{n}{m}=\frac{n}{4}$, we get that $f(W)=\operatorname{Im} f$ and by (C5) we have $f(X) \cap V \subseteq f\left(\left(W \backslash W_{1}\right) \cap X\right)$. 
So that $|f(X) \cap V| \leq 2$, by (3.2). As, by assumption, $v \neq 1$, we obtain easily from (3.3) that either $v=2,3$ or $v=5$. However if $v=3$ or $v=5$ then $X \cap W=\left\{\frac{2 n}{12}, \frac{3 n}{12}, \frac{4 n}{12}\right\}$ or $X \cap W=$ $\left\{\frac{3 n}{20}, \frac{4 n}{20}, \frac{5 n}{20}, \frac{6 n}{20}, \frac{7 n}{20}\right\}$ respectively, so that $X \cap W \subseteq W_{1}$ and thus $f(X) \cap V=\emptyset$, a contradiction. So $v=2$ and hence $f\left(\frac{n}{8}\right)=(\pi, \pi)=f\left(\frac{3 n}{8}\right)$. By condition (C3) $n$ is not a multiple of 8 . Because of (C3) we know that $f\left(\left\lceil\frac{n}{8}\right\rceil\right) \notin V$ and so $f_{j}\left(\left\lceil\frac{n}{8}\right\rceil\right)>\frac{3 \pi}{2}$. Thus

$$
\frac{\pi}{2}<f_{j}\left(\left\lceil\frac{n}{8}\right\rceil\right)-f_{j}\left(\frac{n}{8}\right)=s_{j}(\lceil n / 8\rceil-n / 8) \leq \pi\left(\left\lceil\frac{n}{8}\right\rceil-\frac{n}{8}\right)
$$

and hence $n \equiv q \bmod 8$ with $q=1,2$ or 3 . This implies that for $k=\left\lfloor\frac{n}{8}\right\rfloor$ one has

$$
\frac{5 \pi}{8} \leq \pi-\frac{q \pi}{8}=f_{j}(k)<f_{i}(k)<\pi
$$

and

Consequently,

$$
\frac{\pi}{4}>f_{1}(k)=\frac{2 \pi \frac{n-q}{8}}{n}=\frac{\pi}{4}-\frac{\pi q}{4 n} \geq \frac{\pi}{4}-\frac{3 \pi}{800}=\frac{197 \pi}{800} .
$$

$$
\left|\sin f_{1}(k) \sin \frac{f_{i}(k)}{2} \sin ^{2} \frac{f_{j}(k)}{2}\right| \geq \sin \frac{197 \pi}{800} \sin ^{3} \frac{5 \pi}{8} \geq \frac{1}{4}
$$

and

$$
\left|\sin f_{1}(k) \sin ^{2} \frac{f_{i}(k)}{2} \sin \frac{f_{j}(k)}{2}\right| \geq \sin \frac{197 \pi}{800} \sin ^{3} \frac{5 \pi}{8} \geq \frac{1}{4}
$$

in contradiction with $(\mathrm{C} 1)$.

Third we show $m \neq 3$. Suppose the contrary, that is, assume $m=3$. Let $I=\left[0, \frac{n}{3}\right]$. Then $f(X) \cap V \subseteq f\left(\left[0,\left\lceil\frac{n}{8}\right\rceil\right] \cap X\right)$, by (C5). Note that $\left[0, \frac{n}{8}\right] \cap X=\left\{\frac{k n}{3 v} \mid 1 \leq k \leq \frac{3 v}{8}\right\}$ has $\left\lfloor\frac{3 v}{8}\right\rfloor$ elements. So (3.2) implies

$$
|f(X) \cap V| \leq\left|X \cap\left(0,\left\lceil\frac{n}{8}\right\rceil\right)\right| \leq\left\lfloor\frac{3 v}{8}\right\rfloor+1
$$

Hence a careful analysis of inequality (3.4) and (3.3) yields that $v=9,2,3,5$ or 6 and in all these cases $|f(X) \cap V|>\left\lfloor\frac{3 v}{8}\right\rfloor$. We conclude that $X \cap\left[\frac{n}{8},\left\lceil\frac{n}{8}\right\rceil\right]$ has one element. The latter is $\frac{4 n}{27}$ if $v=9$, $\frac{n}{6}$ if $v=2, \frac{2 n}{9}$ if $v=3, \frac{2 n}{15}$ if $v=5$, and $\frac{3 n}{18}$ if $v=6$. The smallest of the listed numbers is $\frac{2 n}{15}$ and thus we get

$$
\frac{n}{8} \leq \frac{2 n}{15} \leq\left\lceil\frac{n}{8}\right\rceil
$$

Consequently $\frac{n}{120}=\frac{2 n}{15}-\frac{n}{8}<1$. So $n<120$, and this contradicts the assumption that $n>200$.

We conclude that $m \leq 2$. Note that it follows that $f\left(\frac{n}{4}\right) \neq(0,0)$. We claim that $m v$ is not a multiple of 4 for otherwise $f\left(\frac{n}{4}\right) \in f\left(W_{1}\right) \cap V \cap D$; in contradiction with (C4).

Consider the maps $g:[0, n) \rightarrow \mathbb{R}$ and $\bar{g}:[0, n) \rightarrow T$ given by $g(t)=\frac{\theta}{\alpha} t$ and $\bar{g}(t)=(g(t)+$ $2 \pi \mathbb{Z}, g(t)+2 \pi \mathbb{Z})$. So the image of $\bar{g}$ is $D$ and $\bar{g}(t)=f(t)$ for every $t \in X$. Because of condition (C4), one has

$$
\bar{g}\left(X \cap W_{1}\right) \cap V=\emptyset .
$$

Assume now that $\frac{\pi}{2}<\theta<\frac{3 \pi}{2}$. Then, by condition (C4), $\alpha=\frac{n}{m v} \notin W_{1}$ and because $m v>2$, $\alpha<\left\lceil\frac{n}{8}\right\rceil$. Because 4 does not divide $m v$ we get that $m v \geq 9$. The restriction on $\theta$ easily yields that if $W_{1} \cap X$ has at least three elements then $W_{1} \cap X \cap f^{-1}(V) \neq \emptyset$, contradicting (C4). Thus we get $\left|W_{1} \cap X\right| \leq 2$. Hence the length of the interval $W_{1}$ is at most $2 \alpha$ and at least $\frac{n}{4}-2$. Therefore $\frac{2 n}{9} \geq 2 \alpha \geq \frac{n}{4}-2$ and this implies that $n \leq 72$, a contradiction. 
So it remains to deal with the situation $\theta \leq \frac{\pi}{2}$ or $\theta \geq \frac{3 \pi}{2}$. We deal with $\theta \leq \frac{\pi}{2}$ (the other case is dealt with similarly). Clearly then $v \geq 4$. Because 4 does not divide $m v$ it follows that $v \geq 5$ and $v \neq 8$. If $v=6$, then $\frac{n}{3} \in W_{1} \cap X$ and $\bar{g}\left(\frac{n}{3}\right) \in V$; if $v=7$ and $m=1$ then $\frac{2 n}{7} \in W_{1} \cap X$ and $\bar{g}\left(\frac{2 n}{7}\right) \in V$; and if $v=7$ and $m=2$ then $\frac{n}{7} \in W_{1} \cap X$ and $\bar{g}\left(\frac{n}{7}\right) \in V$. These three cases yields to a contradiction with (3.5) so that $v=5$ or $v \geq 9$.

Assume first that $v=5$. If $m=2$, then $2 \alpha=\frac{n}{5} \in W_{1} \cap X$ and $\bar{g}(2 \alpha) \in V$, again a contradiction with (3.5). Thus $m=1$ and $g(\alpha)=\frac{2 \pi}{5}$. Because of (C3) one has $f(\mathbb{Z} \cap I) \cap V=\emptyset$, where $I=\left[\frac{n}{5}, \frac{n}{4}\right]$. Moreover, for every $x \in I, f_{i}(x) \leq f_{j}(x) \leq f_{i}(x)+\frac{\pi}{2}$, that is $f(I)$ is in the strip between the diagonal and the parallel of the diagonal through $A=\left(\frac{\pi}{2}, \pi\right)$. (Note that this last has a continuation in the lower right corner as it is shown in Figure 2.)

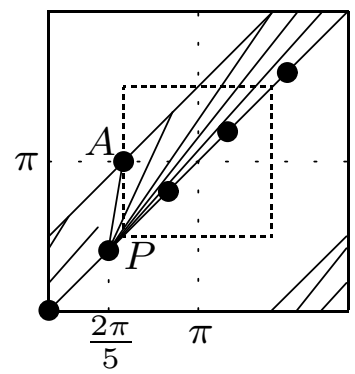

Figure 2

The dots on the diagonal represent the elements of $f(X), f(\alpha)=\left(\frac{2 \pi}{5}, \frac{2 \pi}{5}\right)$ is labelled by $P$. The lines leaving $P$ represent several possibilities for $f(I)$. All of them leave in $P$ and end in the line parallel to the diagonal through $A$. The length of the interval $f_{i}(I)$ is at least $\frac{\pi}{10}$, and the equality holds if and only if $i=1$. If $i=1$ then $f\left(\frac{n}{4}\right)=A$ and $f(I)$ is the segment joining $P$ and $A$. Then $f\left(\left\lceil\frac{n}{4}\right\rceil\right) \in V$ contradicting (C3). Therefore $i \neq 1$ and hence $f(I)$ intersects the interior of $V$ and in fact $f\left[\frac{n}{5}, \frac{9 n}{40}\right]$ intersects $V$. This is because the length of $f_{i}\left[\frac{n}{5}, \frac{9 n}{40}\right]$ is $\frac{\pi i}{20} \geq \frac{\pi}{10}$, and hence there exists $t \in\left[\frac{n}{5}, \frac{9 n}{40}\right]$ with $f_{i}(t)=f_{i}\left(\frac{n}{5}\right)+\frac{\pi}{10}=\frac{\pi}{2}$ and for such a $t$ one has $f(t) \in V$ (look at the picture). Let $t$ be the smallest element of $I$ so that $f(t) \in V$ and set $k=\lfloor t\rfloor$. Then $k+1 \in I$ and hence $f(k+1) \notin V$. This implies that $f_{i}(k+1)>\pi$ (look at Figure 2 ) and therefore $\frac{\pi}{2}<f_{i}(k+1)-f_{i}(k)$, so that the slope of $f_{i}$ is larger than $\frac{\pi}{2}$. Thus

$$
\frac{\pi}{2}=\frac{2 \pi}{5}+\frac{\pi}{10} \leq f_{i}(\lfloor\alpha\rfloor+1) \leq f_{j}(\lfloor\alpha\rfloor+1) \leq \frac{3 \pi}{2}
$$

so that $f(\lfloor\alpha\rfloor+1) \in V$, a contradiction with (C3).

Finally, assume that $v \geq 9$ and recall that we are assuming $\theta \leq \frac{\pi}{2}$. Let $l$ be the first non negative integer such that $g(l \alpha) \geq \pi / 2$ and $p$ the least positive integer such that $g((l+p) \alpha)>3 \pi / 2$. Note that the first inequality implies that $(l-1) \alpha<\frac{n}{4}$. Since $\alpha \leq \frac{n}{9}$ we thus get that $l \alpha \leq \frac{n}{3}<\left\lfloor\frac{3 n}{8}\right\rfloor$ (recall that $n>200$ ). Since $\theta \leq \pi / 2$, we have that $l, p \geq 2$. We claim that $p=2$. Assume that $p \geq 3$. Let $\beta=(l+p-1) \alpha$. Then $\bar{g}(\beta) \in V$ and because of condition (3.5), $\beta \notin W_{1}$. As also, by assumption, $p \geq 3$ and because $g((l+p) \alpha)>\frac{3 \pi}{2}$ it thus follows that $g(\beta)>\frac{3 \pi}{2}-\frac{\pi}{3}=\frac{7 \pi}{6}$. So that the length of $g[0, \beta]$ is at least $\frac{7 \pi}{6}$. If $\beta \leq\left\lceil\frac{n}{8}\right\rceil$, then the length of $g\left(W_{1}\right)$ is at least

$$
\frac{7 \pi}{6} \frac{\left\lfloor\frac{3 n}{8}\right\rfloor-\left\lceil\frac{n}{8}\right\rceil}{\left\lceil\frac{n}{8}\right\rceil} \geq \frac{7 \pi}{3} \frac{n-6}{n+7} \geq 2 \pi .
$$

This implies that $\bar{g}\left(W_{1} \cap X\right) \cap V \neq \emptyset$, contradicting (3.5). So $\beta>\left\lceil\frac{n}{8}\right\rceil$ and hence, as $\beta \notin W_{1}$, we also get $\beta>\left\lfloor\frac{3 n}{8}\right\rfloor$. Let $k$ be the greatest non negative integer such that $(l+k) \alpha \leq\left\lfloor\frac{3 n}{8}\right\rfloor$ (recall 
that $\left.l \alpha<\left\lfloor\frac{3 n}{8}\right\rfloor\right)$. Thus $k<p-1$. As $f((l+k) \alpha) \in V$ and thus $(l+k) \alpha \notin W_{1}$, we obtain that $(l+k) \alpha<\left\lceil\frac{n}{8}\right\rceil$. Consequently,

$$
\left\lfloor\frac{3 n}{8}\right\rfloor<(l+k+1) \alpha \leq 2(l+k) \alpha \leq 2\left\lceil\frac{n}{8}\right\rceil \leq \frac{n+7}{4} \leq\left\lfloor\frac{3 n}{8}\right\rfloor
$$

a contradiction. So indeed $p=2$. Consequently, $\frac{\pi}{2}+3 \theta>\frac{3 \pi}{2}$ and thus $\theta>\frac{\pi}{3}$. Hence, $g(2 \alpha)>$ $\frac{2 \pi}{3}>\frac{\pi}{2}$. Thus $l=2$ and so $\bar{g}(3 \alpha) \in V$, so that $3 \alpha \notin W_{1}$. Since $3 \alpha \leq \frac{n}{3}$ we get $3 \alpha<\left\lceil\frac{n}{8}\right\rceil$. Since also $g(4 \alpha)=4 \theta>\frac{3 \pi}{2}, g(3 \alpha)=3 \theta \geq \frac{9 \pi}{8}$ and we therefore obtain that the length of $g\left(W_{1}\right)$ is at least

$$
\frac{9 \pi}{8} \frac{\left\lfloor\frac{3 n}{8}\right\rfloor-\left\lceil\frac{n}{8}\right\rceil}{\left\lceil\frac{n}{8}\right\rceil} \geq \frac{9 \pi}{4} \frac{n-6}{n+7} \geq 2 \pi .
$$

Consequently, $\bar{g}\left(W_{1} \cap X\right) \cap V \neq \emptyset$ again a contradiction.

This finishes the proof for $v \neq 1$.

Case 2: $v=1$ and $m \geq 3$. Let $\lambda=\frac{i}{m}=\frac{j}{m}-1$, a positive integer. The slope of $f$ is $\frac{j}{i}=1+\frac{1}{\lambda}$. Figure 3 shows the image $f\left[0, \frac{n}{j}\right]$ for $\lambda=1,2$ and 3 . The greater $\lambda$ the closer this image is to the diagonal D.

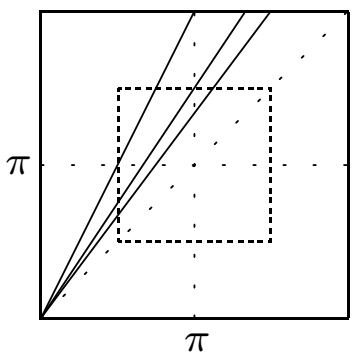

Figure 3

Suppose $m \geq 4$. Let $t_{0}$ be the greatest element of $\left[0, \frac{n}{4}\right] \cap X$ and $t_{1}$ the next element of $X$. Note that $t_{1}-t_{0}=\frac{n}{m} \leq \frac{n}{4}$ and hence $\frac{t_{0}+t_{1}}{2}=t_{0}+\frac{t_{1}-t_{0}}{2} \leq \frac{3 n}{8}$. Clearly $t_{0}=\left\lfloor\frac{m}{4}\right\rfloor \frac{n}{m}$. So if $m \geq 6$ then $t_{0} \geq \frac{m-3}{4} \frac{n}{m} \geq \frac{n}{8}$. On the other hand, if $m=4$ or 5 , then $t_{0}=\frac{n}{m} \geq \frac{n}{5}$. So it follows that for $m \geq 4, I=\left[t_{0}, \frac{t_{0}+t_{1}}{2}\right] \subseteq W$. Because of condition (C3) we thus get $f(I \cap \mathbb{Z}) \cap V=\emptyset$. Set $k=\left\lfloor t_{0}\right\rfloor+1 \in W$. Then $0<f_{j}(k) \leq \pi$ and thus $f_{i}(k)<\frac{\pi}{2}$ (Figure 3 is helpful here). Let $p$ be the least non negative integer so that $f_{i}(k+p) \geq \frac{\pi}{2}$. Since $\pi \in f_{i}(I)$ (as at least half of circle is covered by $\left.f_{i}(I)\right)$, we have that $k+p \in I \cap \mathbb{Z}$ and so $f_{j}(k+p)>\frac{3 \pi}{2}$.

Assume that $\lambda \neq 1$, of the possible lines representing $f\left[0, \frac{n}{j}\right]$ in Figure 3 , the most left one is excluded. Then a look at the picture shows that $f_{j}(k+p)>\frac{3 \pi}{2}$ implies that $f_{i}(k+p)>\pi$. So $s_{i}>\frac{\pi}{2}$. It then follows easily that $p=1$. So $f_{j}(k+1)>\frac{3 \pi}{2}$. Since $s_{j} \leq \pi$ we get that $f_{j}(k)>\frac{\pi}{2}$. A similar backward argument shows that $\pi \leq f_{j}\left(\left\lceil t_{0}\right\rceil-1\right)<\frac{3 \pi}{2}$. Consequently, $\left|f_{j}\left(\left\lfloor t_{0}\right\rfloor+1\right)-f_{j}\left(\left\lceil t_{0}\right\rceil-1\right)\right|>\pi$. Using again that $s_{j} \leq \pi$ we thus get that the length of the interval $\left[\left\lceil t_{0}\right\rceil-1,\left\lfloor t_{0}\right\rfloor+1\right]$ is greater than 1 and hence $t_{0}$ is an integer. Therefore $\frac{\pi}{2}<f_{i}(k+1)-f_{i}(k)=f_{i}\left(t_{0}+1\right)-f_{i}\left(t_{0}\right)=f_{i}(k)<\frac{\pi}{2}$, a contradiction.

Thus $\lambda=1$ and hence $j=2 i$ and $m=i$. Figure 4 represents the image of $f$ and the curves $C_{1}$ and $C_{2}$ defined by the equations $\sin (x / 2) \sin ^{2}(y / 2)=\frac{\sqrt{2}}{4}$ and $\sin ^{2}(x / 2) \sin (y / 2)=\frac{\sqrt{2}}{4}$ respectively. 


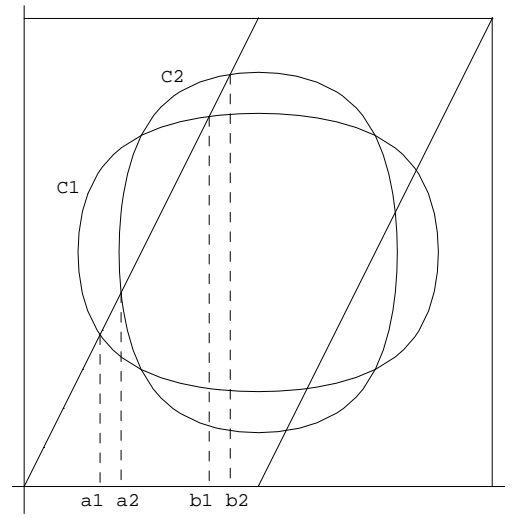

Figure 4

If $(x, y)$ is in the region $R_{1}$ limited by the curve $C_{1}$ then $\sin (x / 2) \sin ^{2}(y / 2) \geq \frac{\sqrt{2}}{4}$ and if $(x, y)$ is in the region $R_{2}$ limited by $C_{2}$ then $\sin ^{2}(x / 2) \sin (y / 2) \geq \frac{\sqrt{2}}{4}$. Let $a_{i}$ and $b_{i}$ be the first coordinates of the intersecting points of the first part of the image of $f$ with $C_{i}(i=1,2)$. Because of condition (C2), there do not exist integers $k_{1}$ and $k_{2}$ in the interval $W$ so that $f\left(k_{i}\right) \in R_{i}$ for both $i=1,2$. Hence it follows easily that $s_{i}>\min \left(b_{1}-a_{1}, b_{2}-a_{2}\right)$. Computing these intersections one obtains that

$$
a_{1} \leq 1.019, \quad a_{2} \leq 1.302, \quad b_{1} \geq 2.484, \quad b_{2} \geq 2.766 .
$$

So $s_{i}>1.464$ and thus $\frac{i}{n}=\frac{S}{2 \pi} \geq 0.233$. As $n>200$ we thus get $i>46$ and hence $\alpha=\frac{n}{m}=$ $\frac{n}{i}<\frac{n}{46}$. It follows that $\frac{n}{4}-\frac{n}{46}<t_{0}<t_{1}<\frac{n}{4}+\frac{n}{46}$. Consequently, for every $t \in\left[t_{0}, t_{1}\right]$ one has $\sin \frac{2 \pi t}{n} \geq \sin \frac{21 \pi}{46}>0.9$. Let $C_{1}^{\prime}$ be the curve defined by the equation $\sin (x / 2) \sin ^{2}(y / 2)=\frac{1}{4 \cdot(0.9)}$ and $C_{2}^{\prime}$ the curve defined by $\sin ^{2}(x / 2) \sin (y / 2)=\frac{1}{4 \cdot(0.9)}$. Let $a_{i}^{\prime}$ and $b_{i}^{\prime}$ be the first components of the intersecting points of $C_{i}^{\prime}$ with the first part of the image of $f$. Then

$$
a_{1}^{\prime} \leq 0.912, \quad a_{2}^{\prime} \leq 1.165, \quad b_{1}^{\prime} \geq 2.573, \quad b_{2}^{\prime} \geq 2.854
$$

Because of condition (C1), one obtains similarly as above that $s_{j}>2 \min \left(b_{1}^{\prime}-a_{1}^{\prime}, b_{2}^{\prime}-a_{2}^{\prime}\right) \geq 3.316>$ $\pi$, a contradiction.

So we have shown that $m=3$. Let $t_{0}=\left\lceil\frac{n}{3}\right\rceil$ and $k=t_{0}-1$. Similarly as in the previous situation (that is, for $m \geq 4$ ), one can now come to a contradiction arguing backward in the interval $\left[\frac{n}{6}, \frac{n}{3}\right]$.

Case 3: $m v \leq 2$. Let $I=\left[t_{0}, 2 t_{0}\right] \cap W$ where $t_{0}=\frac{\alpha}{4}$, so that

$$
f\left(t_{0}\right)= \begin{cases}\left(0, \frac{\pi}{2}\right),\left(\frac{\pi}{2}, \pi\right),\left(\pi, \frac{3 \pi}{2}\right) \text { or }\left(\frac{3 \pi}{2}, 0\right) & \text { if } v=1 \\ \left(\frac{\pi}{4}, \frac{3 \pi}{4}\right),\left(\frac{3 \pi}{4}, \frac{5 \pi}{4}\right),\left(\frac{5 \pi}{4}, \frac{7 \pi}{4}\right) \text { or }\left(7 \frac{\pi}{4}, \frac{\pi}{4}\right) & \text { if } v=2\end{cases}
$$

For every $t \in I$, we have

$$
\frac{\pi}{2} \leq f_{j}(t)-f_{i}(t)=\frac{2 \pi t}{\alpha} \leq \pi
$$

Hence $f(I)$ is in the shadowed part of Figure 5 where the possible values for $f\left(t_{0}\right)$ have been represented by bold circles. 


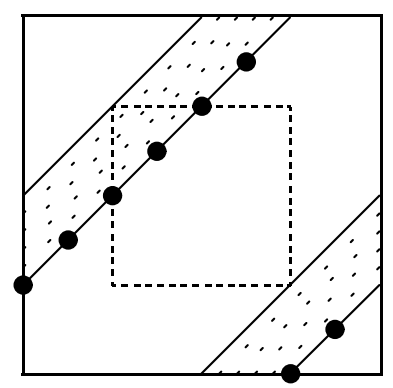

Figure 5

Moreover the slope $s_{j-i}$ of the function $f_{j}-f_{i}$ is $\frac{2 \pi(j-i)}{n}=\frac{2 v m \pi}{n}<\frac{\pi}{50}$ (because by assumption $n>200)$.

The possible values of $m$ and $f\left(t_{0}\right)$ leads to 12 different cases. Using similar arguments one can now show that each of these cases leads to a contradiction, and hence finishes the proof. Crucial in all these arguments is to understand what happens "shortly" after the time $t_{0}$. We illustrate the method for $m=2$ and $f\left(t_{0}\right)=\left(0, \frac{\pi}{2}\right)=O$, so that $v=1$.

Let $k=\left\lceil t_{0}\right\rceil$. As $s_{i} \leq \pi$ and $s_{j-i} \leq \frac{\pi}{50}$ it thus follows that $0 \leq f_{i}(k) \leq \frac{7 \pi}{8}$ and $\frac{\pi}{2}=$ $f_{j}\left(t_{0}\right)-f_{i}\left(t_{0}\right) \leq f_{j}(k)-f_{i}(k)=\left(f_{j}-f_{i}\left(t_{0}\right)\right)+\left(f_{j}-f_{i}\right)\left(k-t_{0}\right) \leq \frac{\pi}{2}+\frac{\pi}{50}=\frac{13 \pi}{25}$. Thus $\frac{\pi}{2} \leq f_{j}(k) \leq$ $\frac{7 \pi}{8}+\frac{13 \pi}{25}<\frac{3 \pi}{2}$. So, because of condition (C3) we obtain that $f_{i}(k)<\frac{\pi}{2}$. Let $l$ be the first integer greater than $t_{0}$ so that $f_{i}(l) \geq \frac{\pi}{2}$. We claim that $f_{i}(l) \geq \frac{3 \pi}{4}$. This is easy if $l \geq \frac{3 n}{16}$, because $m=2$ divides $i$ and then $f_{i}(l) \geq \frac{2 \pi i}{n} \frac{3 n}{16} \geq \frac{3 \pi}{4}$, as desired. So suppose that $l<\frac{3 n}{16}$ and $f_{i}(l)<\frac{3 \pi}{4}$. Then $f_{j}(l)=\left(f_{j}-f_{i}\right)(l)+f_{i}(l)<\frac{4 \pi}{n} \frac{3 n}{16}+\frac{3 \pi}{4}=\frac{3 \pi}{2}$. However this yields a contradiction with condition (C3). This proves the claim $f_{i}(l) \geq \frac{3 \pi}{4}$. Hence it follows that $s_{i} \geq \frac{\pi}{4}$, and thus we get $l \leq k+2$. If the slope of the image of $f$ is $S$, then

$$
\begin{aligned}
1 & <S=\frac{\left(f_{j}(l)-f_{j}\left(t_{0}\right)\right)}{\left(f_{i}(l)-f_{i}\left(t_{0}\right)\right)}=1+\frac{\left(f_{j}(l)-f_{j}\left(t_{0}\right)\right)-\left(f_{i}(l)-f_{i}\left(t_{0}\right)\right)}{\left(f_{i}(l)-f_{i}\left(t_{0}\right)\right)}=1+\frac{s_{j-i}\left(l-t_{0}\right)}{f_{i}(l)} \\
& <1+\frac{\frac{\pi}{50}\left(2+\frac{7}{8}\right)}{\frac{3 \pi}{4}}=\frac{323}{300}=S_{\max } .
\end{aligned}
$$

Let $a$ and $b$ be the first two elements of $I$ so that $A=f(a)=\left(A_{1}, A_{2}\right)$ and $B=f(b)=\left(B_{1}, B_{2}\right)$ belong to the curve $C_{1}$ with equation $\sin (x / 2) \sin ^{2}(y / 2)=\frac{\sqrt{2}}{4}$, and let $c$ and $d$ the first two elements of $I$ so that $C=f(c)=\left(C_{1}, C_{2}\right)$ and $D=f(d)=\left(D_{1}, D_{2}\right)$ belong to the curve $C_{2}$ with equation $\sin ^{2}(x / 2) \sin (y / 2)=\frac{\sqrt{2}}{4}$. Figure 6 represents the curves $C_{1}$ and $C_{2}$ and the lines $L_{1}$ and $L_{2}$ through $O$ of slopes 1 and $S_{\max }$ respectively.

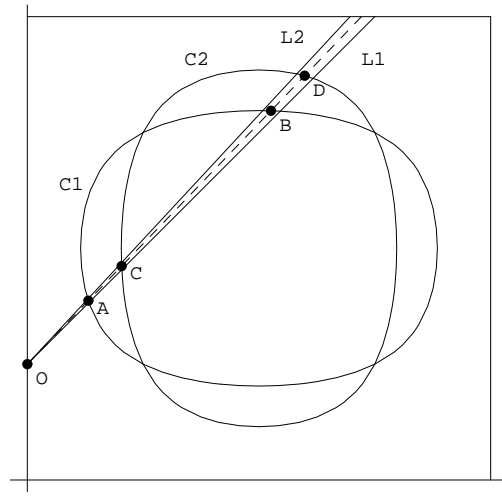

Figure 6 
The image of an interval $\left[t_{0}, t_{0}+\epsilon\right]$ by $f$ lies in the region between $L_{1}$ and $L_{2}$. Computing the intersections of the line through the point $O$ and slopes 1 and $S_{\max }$ one deduces that

$$
\begin{aligned}
& 0.82<A_{1}<0.84 \\
& 1.27<C_{1}<1.29 \\
& 3.19<B_{1}<3.44 \\
& 3.65<D_{1}<3.88
\end{aligned}
$$

Condition (C2) implies that either $f_{i}(l-1)<A_{1}$ and $B_{1}<f_{i}(l)$, or $f_{i}(l-1)<C_{1}$ and $D_{1}<f_{i}(l)$. Thus $l-1-t_{0}=\frac{(l-1)-t_{0}}{l-(l-1)} \leq \max \left\{\frac{A_{1}}{B_{1}-A_{1}}, \frac{C_{1}}{D_{1}-C_{1}}\right\} \leq 0.6$. In particular, $(l-1)-t_{0}<1$. Since also $l, k \in \mathbb{Z}$ and $t_{0} \leq k \leq l-1$ we get that $l=k+1$ and $\left\lceil t_{0}\right\rceil-t_{0}=k-t_{0}<0.6$, so that $n \not \equiv 1,2,3$ $\bmod 8$.

Arguing similarly in the interval $\left[\frac{5 n}{8}, \frac{6 n}{8}\right]$ (note that $f\left(\frac{5 n}{8}\right)=5 f\left(t_{0}\right)=O$ ) one deduces that $5 n \not \equiv 1,2,3 \bmod 8$, so that $n \not \equiv 5,7 \bmod 8$. If $n \equiv 0 \bmod 8$ then $f(l) \in V$ contradicting (C3). We conclude that $n \equiv 4 \bmod 8$ or $n \equiv 6 \bmod 8$.

Condition (C2) implies that either $f_{i}(k)<A_{1}$ and $f_{i}(k+1)>B_{1}$, or $f_{i}(k)<C_{1}$ and $f_{i}(k+1)>$ $D_{1}$. Therefore $s_{i}>\min \left(B_{1}-A_{1}, D_{1}-C_{1}\right) \geq 2.35$.

Assume that $n \equiv 4 \bmod 8$. Then $f_{i}(k)=s_{i} / 2>2.35 / 2>A_{1}$ and hence $f_{i}(k)<C_{1}$ and $f_{i}(k+1)>D_{1}$. The first implies that $s_{i}<2 C_{1}<2.58$ and the second implies that $s_{i}>\frac{2 D_{1}}{3}>2.43$. Therefore, we have

$$
2 \pi+\frac{\pi}{2}<\frac{7 \cdot 2.43}{2}<f_{i}(k+3)=\frac{7 s_{i}}{2}<\frac{7 \cdot 2.58}{2}<2 \pi+\frac{3 \pi}{2}
$$

and

$2 \pi+\frac{\pi}{2}<\frac{7 \cdot 2.43}{2}+\frac{\pi}{2}<f_{i}(k+3)+\frac{\pi}{2}<f_{j}(k+3)<f_{i}(k+3)+\frac{\pi}{2}+\frac{7 \pi}{2} \frac{\pi}{50}<\frac{7 \cdot 2.58}{2}+\frac{\pi}{2}+\frac{7}{2} \frac{\pi}{50}<2 \pi+\frac{3 \pi}{2}$.

So $f(k+3) \in V$ which yields to a contradiction with (C3).

So $n \equiv 6 \bmod 8$. Then $5 s_{i} / 4=f_{i}(k+1)>B_{1}>3.19$, so that $s_{i}>2.55$. Let $h=\left\lceil\frac{n}{4}\right\rceil=\frac{n+2}{4}$. Then $\frac{\pi}{2}<f_{1}(h)=\frac{\pi(n+2)}{2 n}<\frac{\pi}{2}+\frac{\pi}{200}=\frac{102 \pi}{200}, 1.27<s_{i} / 2=f_{i}(h)<\frac{\pi}{2}$ and $\pi<f_{j}(h) \leq f_{i}(h)+\pi+\frac{\pi}{100}$, so that

$$
\begin{aligned}
& \min \left\{\left|\sin f_{1}(h) \sin f_{i}(h / 2) \sin ^{2} f_{j / 2}(h / 2)\right|,\left|\sin f_{1}(h) \sin ^{2} f_{i}(h / 2) \sin f_{j / 2}(h / 2)\right|\right\} \geq \\
& \sin \frac{102 \pi}{200} \sin ^{2} \frac{1.27}{2} \sin \frac{(1.27+101 \pi / 100)}{2}>\frac{1}{4},
\end{aligned}
$$

which yields to a contradiction with $(\mathrm{C} 1)$.

\section{Final Remarks}

From the first part of the paper it is clear that the problem of the freeness of the group generated by two bicyclic units is usually a consequence of a problem of determining when the group generated by two $2 \times 2$-matrices is a free group. In particular, with notation as in Proposition 2.4, to ensure that the group $G=\langle 1+a, 1+b\rangle$ is free of rank 2 it is enough to show that the two matrices

$$
\left(\begin{array}{ll}
1 & 2 \\
0 & 1
\end{array}\right) \quad\left(\begin{array}{ll}
1 & 0 \\
\lambda & 1
\end{array}\right)
$$


generate a free group, where $2 \lambda=\operatorname{tr}(\rho(a b)) \in \mathbb{C}$. If this is the case it is common to say that $\lambda$ is a free point [1]. So far we have used Sanov's Theorem that states that a complex number of modulus at least 2 is a free point. The problem of deciding when a complex number is a free point is an active topic of research. For numerous complex numbers it has been determined whether they are free or not. For an up to date list we refer to [1].

Recently Bamberg [1] has given a family $F$ of polynomials so that a point is free if and only if it is the root of an element of $F$. However, it is very difficult to check if a particular $\lambda$ is root of one of the given polynomials. In particular we don't know if $\sqrt{3}$ is a free point. We now outline that an answer to the latter is needed to deal with bicyclic units of arbitrary dihedral groups.

Let $b_{1}, b_{2}$ be two non commuting bicyclic units of the same type in $\mathbb{Z} D_{n}$. It is again easily seen that we may assume that $b_{1}=\beta_{a^{t}, b}$ and $b_{2}=\beta_{a^{i}, a^{j} b}$. If $x=\left(b_{1}-1\right)\left(b_{2}-1\right)$, then (with the notation of Section 3)

$$
\chi_{k}(x)=16 \sin \frac{2 \pi t k}{n} \sin \frac{2 \pi i k}{n} \sin ^{2} \frac{\pi j k}{n} .
$$

In general it is not true that there is $k$ so that $\chi_{k}(x) \geq 4$. For example, if $n=12, t=j=2$ and $i=3$, then $\chi_{k}(x)=0$ if $k=2,3,4$ and $\left|\chi_{k}(x)\right|=2 \sqrt{3}<4$ if $k=1,5$. Let us look at the representations. The Wedderburn decomposition of $\mathbb{Q} D_{12}$ is well known:

$$
\mathbb{Q} D_{12}=4 \mathbb{Q} \oplus 3 M_{2}(\mathbb{Q}) \oplus M_{2}(\mathbb{Q}(\sqrt{3})) .
$$

Taking appropriate basis one may make the following identifications:

$$
b_{1}=(1,1,1,1, A, A, 1, C) \quad \text { and } \quad b_{2}=(1,1,1,1,1, B, D),
$$

where $A, B \in M_{2}(\mathbb{Q})$ are non-identity matrices so that $(A-1)^{2}=(B-1)^{2}=0$, and

$$
C=\left(\begin{array}{ll}
1 & 2 \\
0 & 1
\end{array}\right) \quad \text { and } \quad D=\left(\begin{array}{cc}
1 & 0 \\
\sqrt{3} & 1
\end{array}\right)
$$

Thus $\left\langle b_{1}, b_{2}\right\rangle$ is free if and only if there do not exist non zero integers $h_{1}, \ldots, h_{m}$ and $k_{1}, \ldots, k_{m}$ so that

$$
h_{1}+\ldots+h_{m}=k_{1}+\ldots+k_{m}=0 \quad \text { and } \quad C^{h_{1}} D^{k_{1}} C^{h_{2}} D^{k_{2}} \ldots C^{h_{m}} D^{k_{m}}=I .
$$

In particular if $\sqrt{3}$ is a free point then $\left\langle b_{1}, b_{2}\right\rangle$ is free.

Actually the previous case seems to be the only problematic case in dihedral groups. Indeed, a computer search for $n \leq 200$ shows that if $n$ is not a multiple of 12 then for every $1 \leq t, i, j<n$ there exists a $k$ so that $\left|\chi_{k}(x)\right| \geq 4$. Moreover if $n=12 m$ then the only values of $(t, i, j)$ for which $\left|\chi_{k}(x)\right|<4$ for every value of $k$ are

$$
(2,3,2) m, \quad(3,2,2) m, \quad(4,3,2) m \quad \text { and } \quad(3,4,2) m .
$$

After an appropriate reduction one can show that all the cases reduce to $n=12$ and $(t, i, j)=$ $(2,3,2)$. This is precisely the example dealt with above. So, this seems to be an indication that for every two non commuting bicyclic units $b_{1}$ and $b_{2}$ of the same type in $\mathbb{Z} D_{n}$ one has that $\left\langle b_{1}, b_{2}^{2}\right\rangle$ is a free group and if $\sqrt{3}$ is a free point then $\left\langle b_{1}, b_{2}\right\rangle$ is always free.

We finish with a remark on the group generated by two bicyclic units of different type. Let $b_{1}=\beta_{a, b}$ and $b_{2}=\gamma_{a^{i}, a^{j} b}$. Then for every irreducible complex character $\chi_{k}$ of $D_{n}$ we get

$$
\chi_{k}(x)=16 \sin \frac{2 \pi k}{n} \sin \frac{2 \pi i k}{n} \cos ^{2} \frac{\pi j k}{n} .
$$


However, for this formula there is no analogue of Lemma 3.1. Indeed, for $n=3$, that is for $D_{3}$, there are pairs of bicyclics with "bad" trace. For example, if $b_{1}=\beta_{a, b}$ and $b_{2}=\gamma_{a, a b}$, then

$$
\chi_{1}(x)=16 \sin \frac{2 \pi}{3} \sin \frac{2 \pi}{3} \cos ^{2} \frac{\pi}{3}=3 .
$$

This implies that $\left\langle b_{1}, b_{2}\right\rangle$ is not free. Actually as mentioned in [2] the group $\left\langle b_{1}, b_{2}\right\rangle$ contains the trivial unit $a \in D_{3}$.

Acknowledgment. We would like to express our gratitude to Victor Jimnez for some helpful conversation on inequality (3.1).

\section{References}

[1] J. Bamberg, Non-free points for groups generated by a pair of $2 \times 2$ matrices, J. London Math. Soc. (2) 62 (2000) 795-801.

[2] A. Dooms and E. Jespers, Normal complements of the trivial units in the unit group of some some integral group rings, preprint.

[3] E. Jespers and G. Leal, Generators of large subgroups of the unit group of integral group rings, Manuscripta Math. 78 (1993), 303-315.

[4] Z.S. Marciniak and S.K. Sehgal, Constructing free subgroups of integral group rings Proc. AMS 125 (1997), 1005-1009.

[5] J. Ritter and S.K. Sehgal. Generators of Subgroups of $U(\mathbb{Z} G)$, Contemporary Math. 93 (198) 331-347.

[6] J. Ritter, S.K. Sehgal, Construction of units in integral group rings of finite nilpotent groups, Trans. Amer. Math. Soc. 324 (2) (1991), 603-621.

[7] A. Salwa, On free subgroups of units of rings, preprint.

[8] I.N. Sanov, A property of a representation of a free group, Doklady Akad. SSSR 57 (1947) $657-659$. 